\title{
A reaction cell for ambient pressure soft x-ray absorption spectroscopy
}

C. Castán-Guerrero, D. Krizmancic, V. Bonanni, R. Edla, A. Deluisa, F. Salvador, G. Rossi, G. Panaccione, and P. Torelli

Citation: Review of Scientific Instruments 89, 054101 (2018); doi: 10.1063/1.5019333

View online: https://doi.org/10.1063/1.5019333

View Table of Contents: http://aip.scitation.org/toc/rsi/89/5

Published by the American Institute of Physics

\section{PHYSICS TODAY}

WHITEPAPERS
MANAGER'S GUIDE

Accelerate R\&D with

Multiphysics Simulation

\section{READ NOW}

PRESENTED BY D $\subset$ MSOL 


\title{
A reaction cell for ambient pressure soft $x$-ray absorption spectroscopy
}

\author{
C. Castán-Guerrero, ${ }^{1}$ D. Krizmancic, ${ }^{1}$ V. Bonanni, ${ }^{1,2}$ R. Edla, ${ }^{1}$ A. Deluisa,${ }^{1}$ F. Salvador,${ }^{1}$ \\ G. Rossi, ${ }^{1,2}$ G. Panaccione ${ }^{1}$ and P. Torelli ${ }^{1}$ \\ ${ }^{1}$ Laboratorio TASC, IOM-CNR, S.S. 14 km 163.5, Basovizza 34149, Trieste, Italy \\ ${ }^{2}$ Università di Milano, Dipartimento di Fisica, Via Celoria 16, I-20133 Milano, Italy
}

(Received 13 December 2017; accepted 15 April 2018; published online 3 May 2018)

\begin{abstract}
We present a new experimental setup for performing X-ray Absorption Spectroscopy (XAS) in the soft $\mathrm{X}$-ray range at ambient pressure. The ambient pressure XAS setup is fully compatible with the ultra high vacuum environment of a synchrotron radiation spectroscopy beamline end station by means of ultrathin $\mathrm{Si}_{3} \mathrm{~N}_{4}$ membranes acting as windows for the X-ray beam and seal of the atmospheric sample environment. The XAS detection is performed in total electron yield (TEY) mode by probing the drain current from the sample with a picoammeter. The high signal/noise ratio achievable in the TEY mode, combined with a continuous scanning of the X-ray energies, makes it possible recording XAS spectra in a few seconds. The first results show the performance of this setup to record fast XAS spectra from sample surfaces exposed at atmospheric pressure, even in the case of highly insulating samples. The use of a permanent magnet inside the reaction cell enables the measurement of X-ray magnetic circular dichroism at ambient pressure. Published by AIP Publishing. https://doi.org/10.1063/1.5019333
\end{abstract}

\section{INTRODUCTION}

The growing interest in operando spectroscopies originates from the request to address in realistic conditions the study of several chemical processes, e.g., synthesis, surface modification/functionalization, catalysis, and corrosions. ${ }^{1,2}$ Operando surface characterization techniques in the soft $\mathrm{X}$-ray range are of prominent interest for addressing the abovementioned phenomena. Similar experiments have been first performed in the hard X-ray domain exploiting the high penetration of the hard X-rays. Bulky, differentially pumped, electron analysers have allowed photoemission (NAPXPSnear ambient pressure X-ray photoemission spectroscopy) to become a powerful method for the investigation of the chemical reactions at surfaces, 3,4 albeit restricted to moderate pressures in the mbar range. The same development was not observed for NEXAFS (Near Edge X-ray Absorption Fine Structure) or X-ray Absorption Spectroscopy (XAS) or X-ray Magnetic Circular Dichroism (XMCD) in the soft $\mathrm{X}$-ray regime despite the large interest in these techniques for studying materials science under ultra high vacuum (UHV) conditions, when surfaces or interfaces are involved. A pioneering setup dedicated to near ambient XAS was realized by Knop-Gericke and co-workers ${ }^{5}$ in the late 90s. Successively XAS in the soft X-ray range has been confined to the mbar pressure range ${ }^{6}$ and, only recently, experimental setups dedicated to Ambient Pressure XAS (AP-XAS) measurements have been realized, favoured by the commercial availability of high quality SiN membranes of few tens of nanometers of thickness. At present, a few experimental setups for performing AP-XAS in the soft X-ray range at ambient pressure are available at synchrotron radiation laboratories. ${ }^{7-11}$

Photoemission and XAS/XMCD are complementary methods that contribute to the characterization of surfaces and nanostructures in experiments carried out in UHV. It is surprising that the development of the ambient-pressure implementation of these techniques has not been synchronous, also considering that XAS in the soft X-ray has several advantages with respect to the NAPXPS: the detection mode can be changed (from total and partial electron yield to transmission or fluorescent photon yield) to obtain different degrees of surface sensitivity; the travel of secondary electrons or photons in the gas is considerably longer than the one of primary photoelectrons measured in XPS and this allows operating NEXAFS effectively at ambient pressure while NAPXPS is still confined to the mbar range. The overall signal is (at least in the case of the electron detection mode) remarkably higher than in photoemission permitting faster data acquisition time. All these reasons make XAS technique extremely efficient in the analysis of the chemical state of the transition metals and of the rare earths that have, respectively, the $\mathrm{L}$ and $\mathrm{M}$ thresholds in the soft $\mathrm{X}$-ray range (i.e., the range accessible by synchrotron radiation beamlines equipped with plane grating monochromators). Moreover, the $\mathrm{K}$ edges of the light elements $(\mathrm{C}, \mathrm{N}, \mathrm{O})$ lie in the soft X-ray energy range. These elements are interesting in a wide range of chemical processes and are not accessible by the hard X-ray beamlines. ${ }^{12,13}$

Here we present our new setup for performing XAS at ambient pressure for the study of chemical reactions at surfaces as implemented at the High Energy branch of the APE beamline (APE-HE) of Nanoscience Foundries and Fine Analysis (NFFA) and Istituto Officina dei Materiali-Italian National Research Council (IOM-CNR) at the Elettra synchrotron radiation facility. ${ }^{14}$ This setup is now available to the Elettra international users as well as to the Trieste-NFFA and NFFA-Europe facilities users.

\section{INSTRUMENTAL DEVELOPMENT}

Performing operando ambient pressure XAS implies to cope with severe experimental constraints. 
The pressure inside the reaction cell must reach 1 bar in order to study a variety of processes and their kinetics in realistic conditions. This has to be compatible with the UHV operation constraint of the X-ray beamline and spectrometer chamber. The acquisition time of one X-ray absorption spectrum must be fast enough to follow the sample changes during operando experiments. This implies the need for developing fast energy scan and acquisition mode. The downtime between UHV experiments and AP operando experiments must be short in order to allow for flexible scheduling of the beamline. The details on how each of these requirements has been addressed can be found in Subsections II A-II C.

\section{A. Beamline and vacuum}

The reactor cell was designed to be hosted inside the UHV end station of APE-HE beamline, which is a typical UHV chamber equipped with an electron energy analyser and a vertical sample manipulator. The energy range of the beamline covers the photon energy range between $200 \mathrm{eV}$ and $1400 \mathrm{eV}$ with variable light polarization (linear $\mathrm{H}$ and $\mathrm{V}$, circular $\mathrm{R}$ and $\mathrm{L}$ ).

A schematic top view of the APE-HE end station together with a 3D model of the operando reactor cell is shown in Fig. 1 [panels (a) and (b)]. As it is visible in the scheme, the cell is introduced in the HE chamber coaxially with the X-ray beam. The reaction cell is mounted on an $\mathrm{x}-\mathrm{y}$ table that allows its movement in the plane perpendicular to the incident beam with $5 \mu \mathrm{m}$ vectorial precision. This allows the alignment of the membrane on to the beam.

The sample surface, inside the cell, sits in the focal point of the beamline. The spot size can vary as a function of photon energy, light polarization, and entry slit aperture, but we can reduce the beam spot down to less than $100 \mu \mathrm{m}$ of diameter by means of a double slit system placed at the entry of the end station.

Two UHV gate valves [labeled as v1 and v2 in Fig. 1(a)] separate the vacuum of the chamber from the one of the beamline. V1 is a conventional gate valve that allows the UHV of the beamline to be maintained, while the HE chamber is vented. $\mathrm{V} 2$ is a gate valve with an X-ray window [see Fig. 1(c)], consisting on an array of $4 \times 4$ membranes of $\mathrm{Si}_{3} \mathrm{~N}_{4}$ of $1 \mathrm{~mm}$ $\times 1 \mathrm{~mm}$ size and $50 \mathrm{~nm}$ thickness each in a square supporting frame of $10 \mathrm{~mm} \times 10 \mathrm{~mm}$ size and $200 \mu \mathrm{m}$ thickness.

The estimated X-ray transmission through the window at V2 is depicted in Fig. 1(c). It accounts for the shadowing effect of the supporting frame of the array of membranes as at the V2 position the beam size exceeds the single membrane "tile." By assuming homogeneous illumination of the membrane array and the transmissivity of the $50 \mathrm{~nm}$ thick $\mathrm{Si}_{3} \mathrm{~N}_{4}$ membrane, we obtained the overall transmission curve [Fig. 1(c)]. The measurements reveal a slightly different value compared to the calculations. The small discrepancy is obviously due to the inhomogeneous distribution of the beam on the array of membranes caused by the $4 \times 4$ array geometry of the X-ray windows which stops the beam in correspondence with the frames of the single $\mathrm{Si}_{3} \mathrm{~N}_{4}$ windows. The two valve-arrangements allow venting the chamber and installing the reactor cell. Once a pressure in the $10^{-5}$ mbar range is reached, V2 is closed and V1 is opened. This allows separating the vacuum of the chamber from the UHV of the beamline, without stopping the X-ray beam. This setup allows replacing the sample in the cell and being ready for a new XAS measurement run within tens of minutes. The disadvantage of X-ray flux reduction is compensated by the flexibility of the system and the uptime of the cell. A second important advantage of this solution is that it protects the UHV of the beamline even in case of damage of the sample cell membrane during the measurements. In fact, the pumping system of the

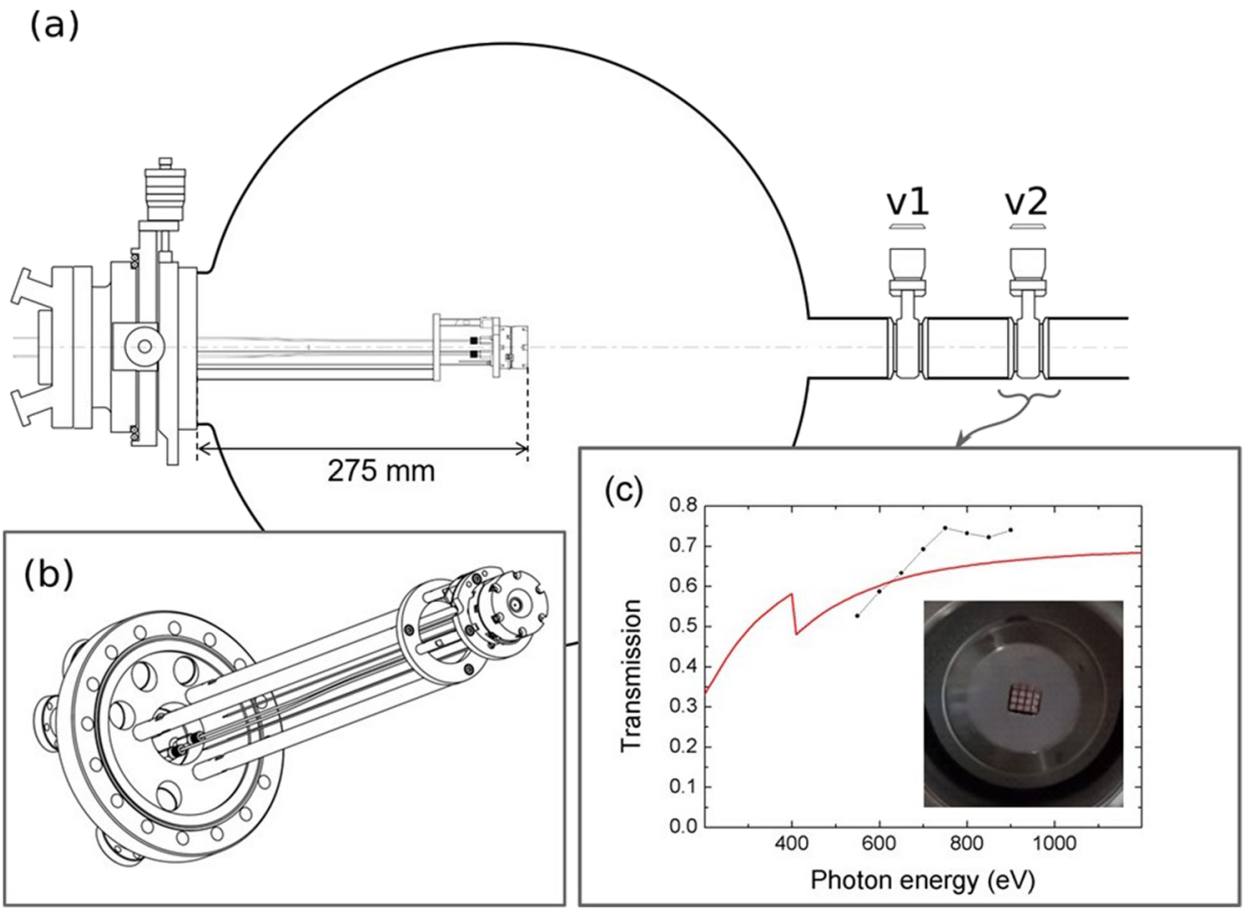

FIG. 1. (a) Scheme of the reactor cell mounting in the HE chamber. (b) $3 \mathrm{D}$ drawing of the reactor cell mounted in the supporting system. (c) Estimated (red curve) and measured (black dots) transmission of the X-ray window mounted on valve $v 2$. In the inset is the real picture of the valve containing the window. 
chamber (two 400-1/min turbo pumps backed by two scroll pumps) is able to preserve a primary vacuum even with a membraneless open cell. The array of membranes that separate the beamline from the sample at AP has already been tested to be robust in an event of non-explosive membrane failure during measurements.

\section{B. Reaction cell design}

A drawing of cross section of the cell and a photo of the reactor cell divided in its constituent parts are shown in Fig. 2.

The main body of the reactor cell consists of an aluminum cylinder containing the sample holder (also made of aluminum), four cylindrical holes for the gas conduits, and an electrical contact (not visible in the picture) for the collection of the total electron yield (TEY) signal by measuring the sample drain current.

The case of the cell is made of polyether ether ketone (PEEK), an UHV compatible, and chemically inert polymer material. $\mathrm{A} \mathrm{Si}_{3} \mathrm{~N}_{4}$ membrane $(500 \mu \mathrm{m} \times 500 \mu \mathrm{m}$ size and $100 \mathrm{~nm}$ thickness in square supporting frames of $10 \mathrm{~mm}$ $\times 10 \mathrm{~mm}$ size and $200 \mu \mathrm{m}$ thickness; Silson, Northampton, UK) is glued to the cell (see Fig. 2; the top cell part) by using silver conductive paste. An electrical contact is also glued to the membrane. The silver paste glue has been chosen as it ensures both electrical conduction and an easy removal in case of membrane replacement. The electrical contact to the membrane allows us to apply a voltage bias between the sample and the membrane. The choice of the $\mathrm{Si}_{3} \mathrm{~N}_{4}$ membrane thickness results from a compromise between high X-ray transparency and mechanical strength. The X-ray transmission through the membrane is above $40 \%$ for the whole available photon energy range and approximately $80 \%$ for photons of $800 \mathrm{eV}$. Regarding the robustness of the membrane, we have verified that it keeps 1 bar differential pressure under beam exposure for several days and after more than 10 venting procedures during the same experiment session.
A molybdenum or kapton spacer is mounted between the PEEK case and the aluminum body. This allows separating the sample from the membrane, forcing the gas to follow a path carved into the spacer. In this geometry, the X-ray beam passes through the membrane and the gas layer and then hits the sample surface and generates the secondary electron emission. The neutralization drain current from the ground is measured through a picoammeter. The length of the X-ray path through the gas can be easily tuned by merely modifying the thickness of the spacer, which is currently of $300 \mu \mathrm{m}$. The sealing is achieved by means of two Viton O-rings located above and below the spacer. Four additional small Viton O-rings seal the cell to the gas circuit. The sealing for the PEEK gas tubes to both the cell and the ConFlat flange consists of commercial F-193 PEEK fittings. ${ }^{15}$ In this setup, we have tested the introduction of gas up to 1 atmosphere of pressure and maintaining the pressure of the UHV chamber in the $10^{-8}$ mbar range.

The temperature of the sample can be controlled between room temperature and $160{ }^{\circ} \mathrm{C}$. The heating is obtained by a cylindrical cartridge heater ( $25 \mathrm{~W}$ of power) inserted in the aluminium body of the cell exactly below the sample. In Fig. 2, the circular hole visible in the aluminium body is where the heater is placed. The temperature is read by a K-type thermocouple in contact with the aluminium body. The maximum achievable temperature is limited to $160{ }^{\circ} \mathrm{C}$ by the two Viton O-rings in the main body of the cell that ensure sealing of the cell with vacuum. The two O-rings are in thermal contact with the aluminium body and therefore they are heated together with the sample and the cell. The performance of the heater has been tested up to $160{ }^{\circ} \mathrm{C}$. The heating process of the cell requires about $20 \mathrm{~min}$ from room temperature to $160^{\circ} \mathrm{C}$; the power needed to maintain the sample at $160^{\circ} \mathrm{C}$ is $6 \mathrm{~W}$. The heater is electrically insulated from the body of the cell; however, leakage currents in the pico-ampere range from the resistance to the cell main body have been observed during the heating process. This leakage introduces a noisy background in the TEY measurements collected from the sample as it is in electrical
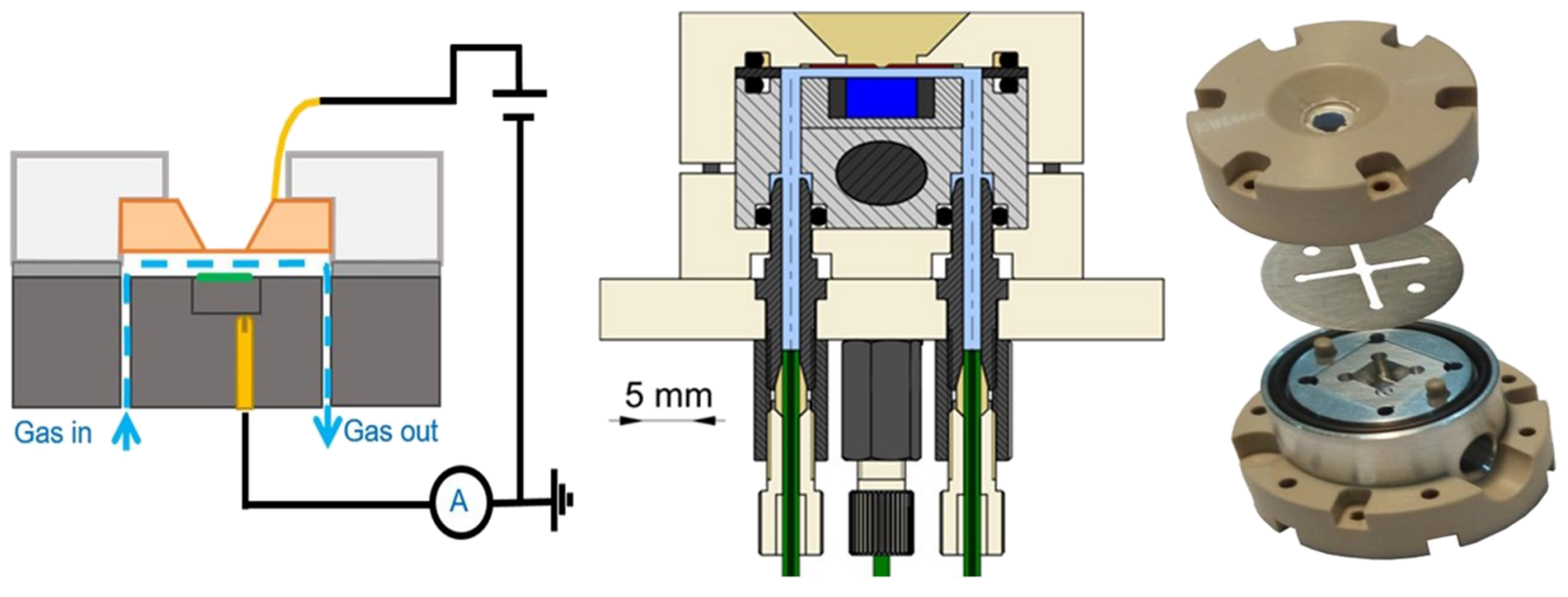

FIG. 2. (Left) Scheme of the electrical circuit of one possible measurement configuration: the drain current is measured from the sample and positive bias voltage is applied to the membrane. (Center) Design of the reaction cell. The different materials are coloured as follows: PEEK in beige and green, Viton O-rings in black, aluminum in grated light gray, stainless steel in grated dark gray, and molybdenum in grated darker gray. The sample position is coloured in blue. (Right) Reaction cell. Top: PEEK cap with silicon nitride membrane. Center: Molybdenum spacer. Bottom: Aluminium main body, with an aluminium sample holder. 


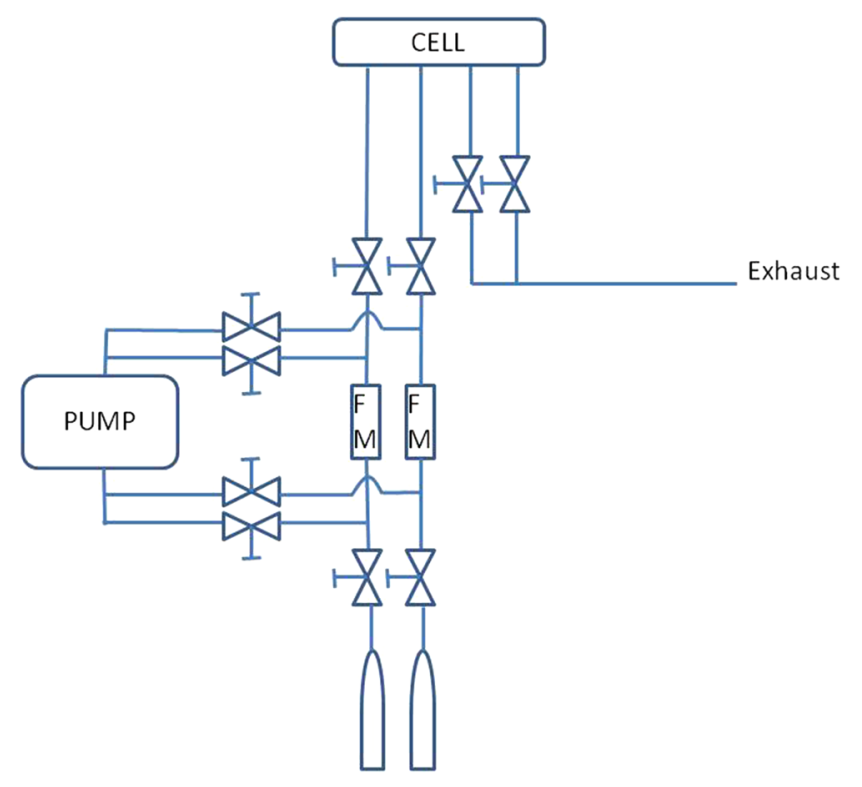

FIG. 3. Scheme of the gas line. The rectangle marked FM are the MKS mass flow meters.

contact with the aluminum body of the cell. Thus to preserve the possibility of measuring the sample current during heating, we have tested an electrical configuration in which the sample was biased at a negative voltage and the TEY signal was recorded on the membrane. This configuration ensured that the sample could be maintained at the wanted temperature without affecting the signal-to-noise ratio of the TEY measurements.

The gas circuit of the reactor cell comprises two inlet gas lines, the tubes which connect the gas lines to the cell, and a small volume in the near sample region where the reaction takes place. Four small ball valves separate the cell circuit (see Fig. 3) from the gas lines, thus making possible to disconnect/connect the cell filled with a desired gas from the gas lines. The gas line system is a homemade gas circuit in which it is possible to connect two gas bottles at a time. The tubes are made of PVC, and two MKS MF1 general mass flow meters control the inlet flux. The exhaust of the gas line after the cell is connected to the gas recuperation unit of the Elettra experimental hall. A pumping stage composed by a membrane pump is connected to the gas lines before and after the flow meters. This pumping stage has a double scope: purging the gas lines and operating the reactor cell in a vacuum. In fact, if the two valves heading to the recuperation line are closed, it is possible to obtain a moderate vacuum in the cell (about $10^{-3} \mathrm{mbar}$ ) that allows recording XAS spectra of the sample in a vacuum. The cell has a small volume (roughly $4 \mathrm{~cm}^{3}$ ); it was verified that a flux of about 5 SCCM is ideal for recording XAS spectra at ambient pressure. Thus, it is possible to use small gas bottles of 11 capacity filled at 12 bars pressure (like the lightcyl SIAD model $)^{16}$ ensuring $40 \mathrm{~h}$ of operation. Using small gas bottles is also favored from the laboratory safety perspective.

\section{Fast data acquisition mode}

The NEXAFS spectra are recorded in the TEY mode: the two electrical contacts allow us to polarize the membrane (positively in order to accelerate the electrons away from the sample) and measure the drain current to the sample through a Keithley 6514 picoammeter. $^{17}$

The absorption spectrum is usually acquired moving the monochromator with a discrete step and recording the TEY intensity at this energy, repeating this operation for the entire range of interest. This procedure is simple and accurate but has the drawback of long acquisition time due to the dead time intrinsic at this procedure. For this reason, a good absorption spectrum of some tenth of $\mathrm{eV}$ lasts several minutes even in the best conditions. These acquisition times are too long if one wants to follow the kinetics of a chemical reaction, thus we have modified the NEXAFS acquisition mode to a continuous recording mode for reducing the acquisition time of more than one order of magnitude. During the continuous or "fast scan" data acquisition mode, the grating monochromator is scanned continuously through the wanted energy range and the picoammeter signal is recorded in the streaming mode. In detail, the monochromator position is read by an optical encoder whose signal is synchronously sampled together with the measured analog signal from Keithley 6514 picoammeter which reads the drain current. A LabVIEW ${ }^{18}$ based software acts as a TCP/IP server between the acquisition computer and the monochromator control.

This scanning mode permits the reduction of the acquisition time of spectra and can be used with the ambient pressure cell but also when measuring the XAS in the UHV configuration of the APE-HE end station being the monochromator setup totally independent from the ambient pressure setup.

\section{TEY MEASURE OF THE X-RAY ABSORPTION IN THE REACTOR CELL}

The description of X-ray absorption spectra in the presence of gas is more complicated than the one in UHV due to the attenuation of the incoming beam by the gas itself (see the Appendix).

Moreover, when measuring the drain current in the reactor cell (both from the sample and the membrane) we need to take into account that several sources of electrons are present due to the absorption of the primary beam by the reactor cell membrane, the reactant gas, and the sample. Figure 4 represents a sketch of the different sources of electrons in our setup. The membrane emits its own TEY signal from both the front surface (in a vacuum) and the back surface (in the reactor cell). These last electrons travel through the gas in the cell and can collide with the gas molecules and eventually unto the sample surface. The X-ray beam absorbed in the gas also creates photoelectrons and ionized molecules. These electrons and ions can collide with the sample surface modifying the effective drain current measured from the sample (I DRAINSample),

$$
\begin{aligned}
\mathrm{I}_{\text {DRAINSample }}= & \mathrm{I}_{\mathrm{TEY}} \text {, Sample }-\mathrm{I}_{\mathrm{TEY}} \text {, membrane }-\mathrm{I}_{\text {electron from gas }} \\
& +\mathrm{I}_{\text {ions from gas }} .
\end{aligned}
$$

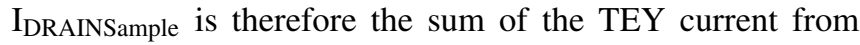
the sample ( $\mathrm{I}_{\mathrm{TEY}}$, Sample $)$ and additional neutralization currents originating from the membrane $\left(\mathrm{I}_{\mathrm{TEY}}\right.$, membrane, from the electrons gas ( $\mathrm{I}_{\text {electrons from gas }}$ ), and from a positive ion current due to the ionized gas atoms ( $\left.\mathrm{I}_{\text {ions from gas }}\right)$ [Eq. (1)]. 

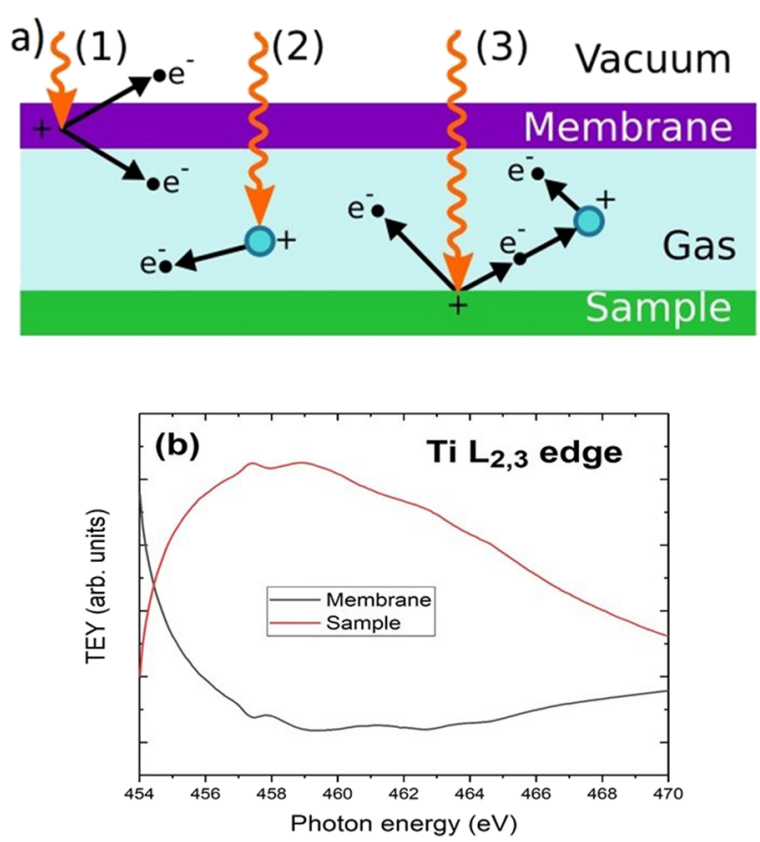

FIG. 4. (a) Scheme of all the electron and ion yield channels originated by photons hitting (1) the membrane, (2) the gas, and (3) the sample. (b) TEY spectrum of the Ti $\mathrm{L}_{2,3}$ edge of a titanium foil inside the reactor with a flux of helium at 1 bar. Red and black lines show the spectrum is acquired from the electrical contact of the sample and membrane, respectively, while the other contact was grounded.

The additional terms to $\mathrm{I}_{\mathrm{TEY}}$, Sample can be considered constant as due to constant cross sections, assuming that there are no absorption edges of elements present in the membrane or in the gas in the scanned energy range. Thus, they should not modify the spectral line shape of the sample.

Moreover the photoemitted electrons (from sample, gas, and membrane) can further ionize the gas creating an avalanche of low energy electrons. This effect obviously depends on the ionization energy of the gas, the gas pressure, and the energy of the photoemitted electrons and it was described by Townsend early in $1900 .{ }^{19}$ This contribution can dominate the TEY signal when operating at high gas pressure and can be varied playing with the gaseous species and the application of an accelerating electric field.

Due to the many sources of the TEY signal in the reactor cell and to the avalanche effect, the XAS spectra at ambient pressure can present high background levels with non-linear shapes that can represent a problem in the data analysis. For these reasons, the TEY measurement accuracy requires more careful evaluation in a reactor cell compared to the UHV case.

The fluorescence yield consequent to X-ray absorption can also be absorbed by the gases and by the membrane creating photoelectron currents that also contribute to the measured TEY. We will show below that this contribution, at soft X-ray energies, can be neglected.

In our setup, the membrane and the sample are electrically isolated, allowing for different measurement configurations (i.e., biasing sample or membrane and simultaneously recording two currents). The $\mathrm{L}_{2,3}$ XAS spectrum of Ti from a titanium foil, collected simultaneously from the membrane and the sample drain currents, is shown in Fig. 4(b). The spectrum is obtained from a Ti foil non-atomically cleaned and exposed to 1 bar of pure He pressure during the data acquisition, with no applied bias voltage. The signals as recorded from the sample and from the membrane are mirror-like, with opposite sign. This indicates that, in our setup, there is a capacitive coupling of the two surfaces and that positive and negative charges created by the photocurrent from the sample surface do neutralize on the opposite electrodes (the membrane and the sample).

\section{EXPERIMENTAL RESULTS}

In this section, we will present XAS spectra acquired reading the drain current on the sample while applying a positive bias potential to the $\mathrm{SiN}$ membrane. Indeed the application of a positive bias to the membrane helps us to extract the electrons from the sample surface, as it happens also in UHV. In the presence of high gas pressure, this is particularly important because it favours the creation of a Townsend avalanche thus improving considerably the signal over background ratio.

The full electrical polarization of the SiN membrane could be questionable due to its insulating nature. However, the small dimension of the window and its integration in a semiconducting silicon wafer allow setting an electric field on the sample surface simply by polarizing the Si wafer. Moreover, during measurements the $\mathrm{SiN}$ membrane is illuminated by an intense X-ray beam that reduces its resistivity thus increasing the charges' mobility.

Figure 5 displays several XAS spectra acquired measuring the sample drain current, with bias voltages from $-30 \mathrm{~V}$ to $+30 \mathrm{~V}$ applied to the membrane. The sample is a pressed $\mathrm{CeO}_{2}$ powder (glued with conductive paste on the aluminum sample holder). The spectra were acquired in $1 \mathrm{bar}$ of $\mathrm{He}$ on the $\mathrm{Ce}_{4,5}$ absorption edges (about $900 \mathrm{eV}$ ). The $\mathrm{CeO}_{2} \mathrm{M}_{4,5}$ absorption spectrum displays two intense peaks followed by a satellite at higher photon energies ${ }^{20}$ corresponding to a pure $\mathrm{Ce}^{+4}$ oxidation state.

The sample signal increases for increasing bias voltage due to the creation of a larger avalanche as a function of the applied acceleration potential. Thus the ionization of the gas molecules by the photoemitted current greatly affects the TEY giving the possibility to increase measured current by applying an optimal bias voltage, i.e., exploiting the gas as a primary electron multiplier. In order to evaluate the advantage of the biased configuration, we evaluated the signal/background ratio [Fig. 5, panel (b)]. The results show a gain in the quality of the spectra when biasing with a few volts and that saturates at approximately $5 \mathrm{~V}$. The saturation bias depends on the gas according to its ionization energy.

To investigate the effect of different gases on the TEY signal, we recorded the $\mathrm{Fe} \mathrm{L}_{2,3}$ edge from an iron oxide sample in 1 bar pressure of a gas mixture, ranging from pure $\mathrm{He}$ to pure nitrogen through different intermediate concentrations. These spectra, recorded with a fixed bias voltage of $20 \mathrm{~V}$, are presented in Fig. 5 [panel (c)]. It appears that the XAS peak intensity is reduced by a factor 2 when going from pure helium to pure nitrogen with a simultaneous increase of the background by a factor 10 . These two effects concur in making the spectrum recorded in helium of a better quality than the one recorded in nitrogen. The background increase is due 

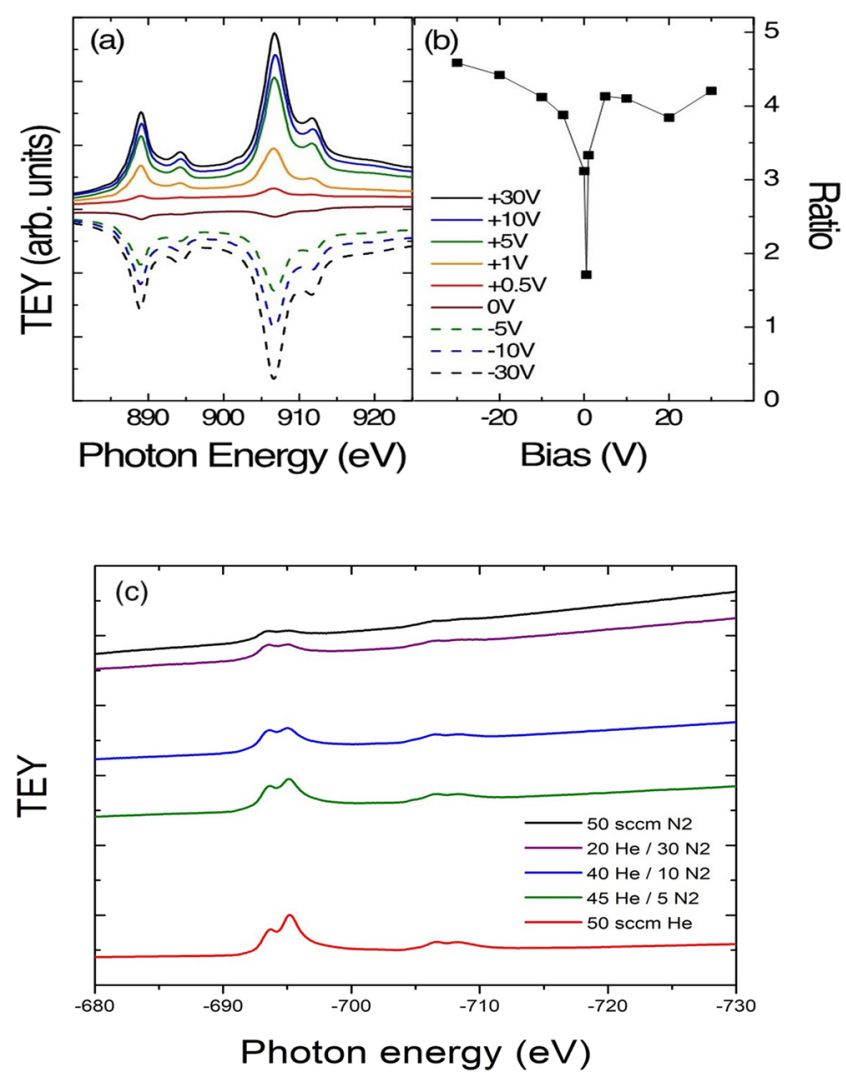

FIG. 5. (a) XAS spectrum of the $\mathrm{Ce}_{4,5}$ absorption edge in ceria powder as a function of the bias voltage. (b) Ratio between the XAS signal at the $\mathrm{M}_{4}$ peak and in the background, as a function of the bias voltage. (c) Fe $\mathrm{L}_{2,3} \mathrm{XAS}$ spectra from iron oxide taken in mixtures of $\mathrm{N}_{2}$ and $\mathrm{He}$ going from pure $\mathrm{He}$ to pure $\mathrm{N}_{2}$.

to the absorption of the X-ray beam that is higher in $\mathrm{N}_{2}$ than in $\mathrm{He}$, thus creating photoelectrons and ions in the gas volume. The reduction of the edge jump is largely due to the different efficiency in the avalanche creation of $\mathrm{He}$ and $\mathrm{N}_{2}$. This last effect can be tackled by increasing the applied bias. However the increased potential that pushes away the electrons from the sample surface attracts the positive gas ion at the same time. These ions bombard the surface releasing an energy equal to the applied potential. Therefore, the application of a high voltage potential between the membrane and the sample can also lead to a degradation of the sample surface over the measuring time. The observation of the signal variability as a function of the relative gas concentration involves an important warning: if relative gas concentration is quickly varied during the acquisition of a spectrum, it is possible that it results in a distortion of the XAS features. For this reason, great care in the data analysis of the spectra acquired immediately after or during the gas introduction must be made keeping in mind that the electron yield is affected by the gas composition.

We have decided to analyze the fluorescence contribution to our spectra to rule out the possibility that at ambient pressure and in our particular setup the fluorescence yield is enhanced with respect to the UHV case. To experimentally estimate the contribution due to the fluorescence, we measured a capped sample $-2 \mathrm{~nm} \mathrm{MgO} / 30 \mathrm{~nm} \mathrm{Fe} / 100 \mathrm{~nm} \mathrm{BaTiO}_{3} / \mathrm{SrTiO}_{3}$ bulkin the reactor cell at 1 bar pressure of pure He. The typical shape of the $\mathrm{L}_{2,3}$ absorption edges of metallic Fe has been collected, similar to the spectra recorded in UHV experiments. On this same sample we attempted to measure the $\mathrm{Ti}_{2,3}$ edge, but no signal was detectable. This test rules out the possibility that fluorescence significantly contributes to the measured signals in this energy range as the probing depth of the fluorescence photons is roughly two orders of magnitude larger than the probing depth of TEY. These findings confirm that in our system the TEY is dominated by the Auger decay as expected: in the soft $X$-ray range and for the materials we study (low atomic number $\mathrm{Z}$ ), the Auger electron yield is favoured compared to the fluorescence yield by about two orders of magnitude. ${ }^{21}$

One remarkable result of the TEY measurements in our gas reactor is that it is possible to perform experiments even on highly insulating samples, which are impossible to measure in a vacuum. The XAS spectrum of the Ce $\mathrm{M}_{4,5}$ absorption edge in Fig. 6 (top panel) was measured from a sample of $\mathrm{CeO}_{2}$ impregnated on a highly insulating $2 \mathrm{~mm}$ thick yttria-stabilized zirconia (YSZ) substrate. In UHV, this sample could not be measured due to electric charging, but inside the reactor, under a flux of $50 \mathrm{SCCM}$ of $\mathrm{He}$ at 1 bar, we obtained a high statistics spectrum in just $1 \mathrm{~min}$. The good signal over noise ratio motivated us to test the limit of the spectrum acquisition speed in view of possible applications in the study of the reaction kinetics. In Fig. 6 (bottom panel),
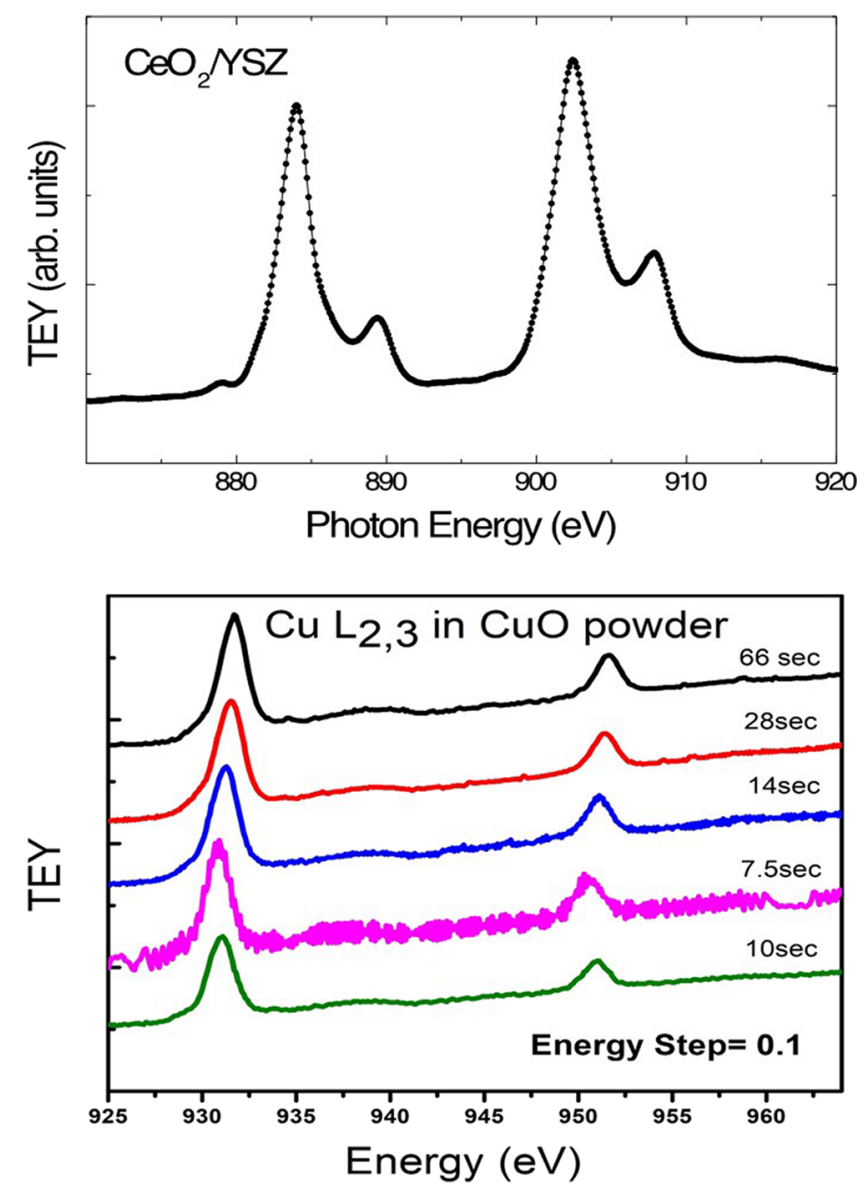

FIG. 6. Top: XAS spectrum of the $\mathrm{Ce}_{4.5}$ absorption edge in $\mathrm{CeO}_{2} / \mathrm{YSZ}$, with a bias voltage on the membrane of $+20 \mathrm{~V}$ and a He flux of $50 \mathrm{SCCM}$ at 1 bar, using the fast data acquisition mode. Bottom: XAS spectra of the $\mathrm{L}_{2,3}$ edge of $\mathrm{Cu}$ from $\mathrm{CuO}$ powder recorded in $1 \mathrm{bar}$ of $\mathrm{He}$ at different acquisition speeds. 


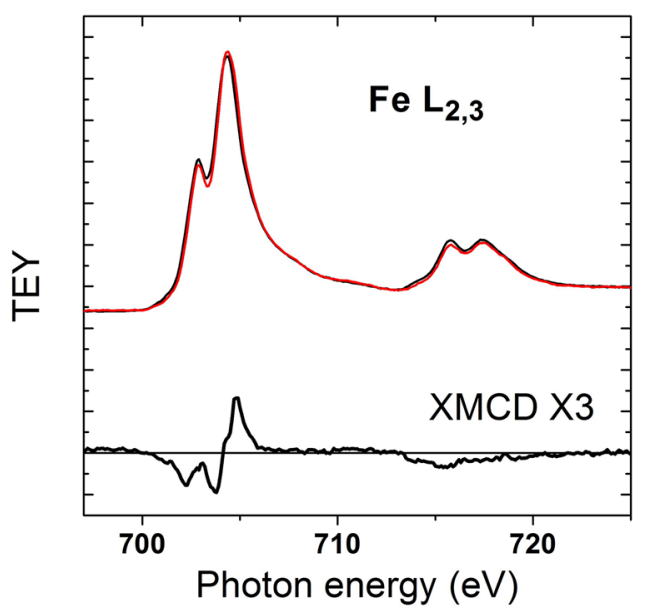

FIG. 7. $\mathrm{XMCD}$ in $\mathrm{Fe}$, in a Fe based permanent magnet inside the cell, with a bias voltage on the membrane of $+20 \mathrm{~V}$ and a 50 SCCM He flux at 1 bar.

we show the spectra of the $\mathrm{L}_{2,3}$ edge of $\mathrm{Cu}$ from $\mathrm{CuO}$ powder recorded in 1 bar of $\mathrm{He}$ at different acquisition speeds. All the spectra are measured in the same energy range of $40 \mathrm{eV}$, with a step size of $0.1 \mathrm{eV}$. It results that the acquisition time can be reduced down to $10 \mathrm{~s}$ without observing distortions in the spectral features. This test indicates that our maximum speed is $4 \mathrm{eV} / \mathrm{s}$, meaning that if the energy range to be explored is limited to $10-20 \mathrm{eV}$, it will be possible to obtain a spectrum in a few seconds.

We have also performed XMCD in the reactor cell by introducing a permanent magnet with the magnetization direction perpendicular to the photons' propagation direction. As shown in Fig. 7, two spectra were recorded under a flux of $50 \mathrm{SCCM}$ of helium at 1 bar with opposite circular polarization in order to obtain the XMCD spectrum. The spectrum line shapes are similar to the pure $\mathrm{Fe}_{3} \mathrm{O}_{4}{ }^{22,23}$ with a more pronounced pre-peak on the $\mathrm{L}_{3}$ edge when compared to $\mathrm{Fe}_{3} \mathrm{O}_{4}$ that makes the spectrum also similar to the maghemite $\left(\mathrm{Fe}_{2} \mathrm{O}_{3}{ }^{24}\right)$ spectrum. This difference is attributed to surface contamination as the sample was measured without any previous surface treatment. This implies that the surface stoichiometry is not well defined and probably many oxides and hydroxide species coexist at the surface and contribute to the spectrum. This explains also the shape of the XMCD signal, which does not coincide with that of $\mathrm{Fe}_{3} \mathrm{O}_{4}$ or $\mathrm{Fe}_{2} \mathrm{O}_{3}$. These data demonstrate that with our AP setup it is possible to measure XMCD magnetic signals from surfaces exposed to 1 bar of gas. This could find interesting applications in the study of molecular magnets and in devices based on magnetic components.

\section{CONCLUSIONS}

We presented a new setup for performing AP-XAS at the APE-HE beamline that is open to users through the NFFA facility. The setup allows for the acquisition of XAS spectra with a good signal-to-noise ratio from samples that are exposed to a flowing gas pressure of 1 bar. The reactor cell has been employed with different gases and gas mixtures. The TEY signal measured at ambient pressure has been analyzed and the route to its optimization for given sample and environmental gas conditions has been identified. The combination of the UHV compatible ambient pressure cell setup, vacuum decoupling highly soft X-ray transparent windows, fast energy scanning, and TEY acquisition mode of near edge XAS creates favourable conditions for surface chemistry studies in the presence of gaseous reagents at atmospheric pressure, at variable substrate temperature and with applied magnetic field. We have also shown that the electron and ion avalanche that is formed in the gas makes it possible to measure XAS spectra of highly insulating samples. This feature is very attractive for surface catalysis studies as many catalysts are highly insulating compounds or are dispersed/supported on highly insulating substrates and can hardly be studied for UHV compatible Langmuir range gas exposures. Operando studies in the setup can be performed by varying the gas composition and pressure and this makes it possible to extend all the power of XAS/XMCD with a variable polarization synchrotron radiation source to the domain of surface chemistry in realistic conditions.

\section{ACKNOWLEDGMENTS}

This work has been performed in the framework of the nanoscience foundry and fine analysis (NFFA-MIUR Italy Progetti Internazionali) project. The facility is offered to users via www.trieste.nffa.eu.

\section{APPENDIX: X-RAY ABSORPTION IN PRESENCE OF A GAS}

X-ray absorption by solids can be measured via the decay signals of the X-ray excited core holes, i.e., by detecting the fluorescence yield and/or the TEY. At soft X-ray energies, the core hole decay is dominated by Auger electron emission. The photo-current emitted from the sample is thus dominated by the primary and Auger electrons plus the avalanche of secondary electrons created by both the primary and the Auger ejected electrons on their scattered way through the surface and vacuum. The detection of TEY, or quantum yield, can be performed on both the ejected electrons, by means of a polarized electron multiplier collecting the electrons in a vacuum or by measuring the neutralization (drain) current by the ground through a picoammeter. TEY is directly proportional to the creation of primary core holes, i.e., to the X-ray absorption cross section. The detection drain current from the sample can be favourable as compared to the detection of ejected electron currents in a vacuum by means of polarized electron multipliers due the simplicity of the experimental apparatus and its viability in the presence of high gas pressure. It only requires a good sample insulation from the ground and a low noise shielded wiring to the picoammeter. This is definitely the only TEY measurement compatible with a high gas pressure.

The absorbed intensity $\left(\mathrm{I}_{\mathrm{a}}\right)$ is the difference between the incident photon flux $\left(\mathrm{I}_{0}\right)$ and the transmitted $\left(\mathrm{I}_{\mathrm{t}}\right)$ one and can be written as ${ }^{25}$

$$
\mathrm{I}_{\mathrm{a}}=\mathrm{I}_{0}-\mathrm{I}_{\mathrm{t}} \approx \mathrm{I}_{0}(\mathrm{E}) \rho_{\mathrm{a}} \mathrm{d} \sigma^{\mathrm{abs}}(\mathrm{E}),
$$

where $\rho_{\mathrm{a}}$ is the atomic density, $\mathrm{d}$ is the sample thickness, and $\sigma^{\text {abs }}(\mathrm{E})$ is the total photoionization cross section at a given 
value of X-ray energy. $\sigma^{\mathrm{abs}}(\mathrm{E})$ depends on the incident energy and reflects the electronic structure of the studied material. Both $\rho_{\mathrm{a}}$ and $\mathrm{d}$ are constant as a function of energy. The incoming $\mathrm{I}_{0}$ changes, on the other hand, as a function of the incident energy and needs to be carefully measured when performing XAS in UHV. This is a first important difference between the TEY measured in UHV and in the presence of high pressure of gas: in fact in the last configuration it is not possible to acquire an $\mathrm{I}_{0}$.

In UHV it is possible to measure the photon flux impinging on the sample surface (for example, by measuring the TEY from a highly transparent metal grid intercepting a fraction of the incoming beam typically before the entrance of the experimental chamber). The same measure is not possible when performing XAS at high pressure due to the presence of the cell membrane window and the gas layer which both act as a photon absorber and modify the $\mathrm{I}_{0}$. Thus in the reactor cell we define the photon flux hitting the sample as $\mathrm{I}_{0 \text { eff }}$,

$$
\mathrm{I}_{0 \text { eff }}=\mathrm{I}_{0} \mathrm{e}^{-\left(\mu_{\text {window }} \times 1_{\text {window }}+\mu_{\text {gas }} \times l_{\text {gas }}\right)},
$$

where $\mu_{\text {window }}$ and $l_{\text {window }}$ together with $\mu_{\text {gas }}$ and $1_{\text {gas }}$ are the $\mathrm{X}$-ray attenuation lengths and the thicknesses of the membrane and the gas, respectively.

$\mathrm{I}_{0}$ eff cannot be directly measured. Consequently the XAS measurements are possible only for those elements that are not present in the gas or in the membrane (e.g., in our system, $\mathrm{Si}$ and $\mathrm{N}$ must be excluded because they are constituents of the membrane).

${ }^{1}$ J. R. Morris, J. N. Russell, Jr., and C. J. Karwacki, J. Phys. Chem. Lett. 6, 4923-4926 (2015).

${ }^{2}$ J. Dou, Z. Sun, A. A. Opalade, N. Wang, W. Fu, and F. Tao, Chem. Soc. Rev. 46, 2001-2027 (2017).
${ }^{3}$ M. Salmeron and R. Scholgl, Surf. Sci. Rep. 63, 169-199 (2008).

${ }^{4}$ D. Pierucci, J. J. Gallet, F. Bournel et al., J. Phys. Chem. C 120, 21631 (2016).

${ }^{5}$ A. Knop-Gericke, M. Havecker, T. Neisius, and Th. Schedel-Niedrig, Nucl. Instrum. Methods Phys. Res., Sect. A 406, 311-322 (1998).

${ }^{6}$ M. Havecker, A. Knop-Gericke, and T. Schedel-Niedrig, Angew. Chem., Int. Ed. 37, 1939 (1998).

${ }^{7}$ P. T. Kristiansen, T. R. C. Rocha, A. Knop-Gericke, J. H. Guo, and L. C. Duda, Rev. Sci. Instrum. 84, 113107 (2013).

${ }^{8}$ Y. Tamenori, J. Synchrotron Radiat. 20, 419-425 (2013).

${ }^{9}$ J. Forsberg, L. C. Duda, A. Olsson et al., Rev. Sci. Instrum. 78, 083110 (2007).

${ }^{10}$ C. Escudero, P. Jiang, E. Pach et al., J. Synchrotron Radiat. 20, 504-508 (2013).

${ }^{11}$ J. J. Velasco-Velez, C. H. Wu, B. Y. Wang, Y. Sun, Y. Zhang, J.-H. Guo, and M. Salmeron, J. Phys. Chem. C 118, 25456-25459 (2014).

${ }^{12}$ C. Lamberti, C. Prestipino, F. Bonino et al., Angew. Chem., Int. Ed. 41, 2341-2344 (2002).

${ }^{13}$ S. Bordiga, E. Groppo, G. Agostini, J. A. van Bokhoven, and C. Lamberti, Chem. Rev. 113, 1736-1850 (2013).

${ }^{14}$ G. Panaccione, I. Vobornik, J. Fujii et al., Rev. Sci. Instrum. 80, 043105 (2009).

${ }^{15}$ IDEX Health \& Science, LLC., 619 Oak Street, Oak Harbor, WA 98277, USA.

${ }^{16}$ See www.siad.com for information about lightcyl SIAD gas bottles.

${ }^{17}$ See www.tek.com/keithley for information about Keithley 6514 picoammeter.

${ }^{18}$ See www.ni.com for information about LABVIEW program.

${ }^{19}$ J. S. Townsend, The Theory of Ionisation of Gases by Collision (London Constable and Company, 1910).

${ }^{20}$ T. Skala, N. Tsud, M. A. Orti, T. O. Mentes, A. Locatelli, K. C. Prince, and V. Matolin, Phys. Chem. Chem. Phys. 13, 7083-7089 (2011).

${ }^{21}$ J. Stöhr, NEXAFS Spectroscopy (Springer, Heidelberg, 1992).

${ }^{22}$ P. Kuiper, B. G. Searle, L. C. Duda, R. M. Wolf, and P. J. van der Zaag, J. Electron Spectrosc. Relat. Phenom. 86, 107-113 (1997).

${ }^{23}$ D. J. Huang, C. F. Chang, H. T. Jeng et al., Phys. Rev. Lett. 93, 077204 (2004).

${ }^{24}$ S. Brice-Profeta, M. A. Arrio, E. Tronc et al., J. Magn. Magn. Mater. 288, 354-365 (2005).

${ }^{25} \mathrm{~J}$. Stöhr and H. C. Siegmann, Magnetism: From Fundamentals to Nanoscale Dynamics (Springer-Verlag, Berlin, 2006). 